\title{
Comunicação
}

\section{Avaliação do papel do cirurgião no tratamento do Tumor de Wilms: análise de um estudo cooperativo}

\author{
S.T. SCHETTINI
}

Disciplina de Cirurgia Pediátrica, Departamento de Cirurgia, UNIFESP - Escola Paulista de Medicina, São Paulo, SP.

RESUMO - Ов EтIvo. Detectar os efeitos do tratamento e estadiamento cirúrgicos nos resultados obtidos e verificar a possível relação entre tais resultados e o cumprimento das normas do protocolo cirúrgico preestabelecido (segundo as normas do NWTS).

Casuística e método. A casuística é composta de 166 pacientes operados, registrados entre outubro de 1986 e dezembro de 1988, com dados atualizados até fevereiro de 1992 . O período mínimo de acompanhamento foi de 24 meses para 147 pacientes e de três a 18 meses para 19 pacientes, sendo o período médio de acompanhamento de 36 meses. A todas as instituições que se integraram ao estudo cooperativo do GCBTTW foram fornecidos os protocolos, visando à uniformização dos procedimentos clínicos e cirúrgicos.

Resultados. Após submeter os dados obtidos à análise estatística, verificamos que:

1) Não existe interferência da ligadura prévia do pedículo nos resultados (recidivas e mortalida-

\section{NTRODUÇÃO}

O Tumor de Wilms constitui um dos primeiros exemplos de êxito no tratamento de neoplasia maligna. Apesar de se tratar de uma neo- plasia com capacidade e vel oci dade de di ssemi nação inferiores à da maioria dos tumores malignos da infância, pode-se afirmar que o pioneirismo do sucesso terapêutico não se deveu a essa relativa menor malignidade, mas foi sim fruto da formação de um grupo cooperativo multidisciplinar e multiinstitucional, o National Wilms Tumor Study -NWT, criado em 1969.

Basicamente, o objetivo foi unir casuísticas de diferentes instituições para obter número suficiente de casos, avaliados e tratados de forma padronizada, permitindo, assim, a obtenção de conclusões cientificamente significativas.

Com esses mesmos objetivos, em 1986 foi formado o Grupo Brasileiro para o Tratamento do Tumor de Wilms (GCBTTW) e instituídos os comitês das diferentes especialidades envolvidas em de); 2) A ruptura tumoral intra-operatória com contaminação da cavidade peritoneal interfere desfavoravelmente no índice de mortalidade. 3) Existe discordância significante entre a opinião do cirurgião e a análise histopatológica quanto à invasão da adrenal e da gordura peri-renal. 4) Os tumores com peso superior a $500 \mathrm{~g}$ têm pior prognóstico. 5) 0 desempenho dos cirurgiões foi consi derado inadequado em relação ao inventário ganglionar (56,6\% dos pacientes não tiveram gângli os analisados), o que pode ter contribuído para: menor índice de recidivas nos pacientes com gânglios acometi dos ; maior incidência de metástases pulmonares nos pacientes sem gânglios analisados; pacientes no Estádio II com melhor prognóstico em relação ao Estádio I, e pacientes em estádios I e III com índices de recidivas semelhantes.

UNITERMOS: Tumor de Wilms. Nefroblastoma. Tratamento cirúrgico.

sua avaliação, tratamento e acompanhamento, aos quais coube a uniformização necessária em sua área de atuação, sob a forma de um protocolo.

Coube-nos a elaboração do protocol o de cirurgia, o qual constituiu um verdadei ro manual de procedimentos quanto às recomendações para a atuação do cirurgião nos portadores do Tumor de Wilms, seguindo-se as recomendações do NWTS .

N osso objetivo no presente estudo é analisar os possíveis efeitos do tratamento e do estadiamento cirúrgico nos resultados obtidos no tratamento dos pacientes do GCBTTW, assim como a possível relação de tais resultados com o cumprimento das recomendações estabel ecidas no protocolo cirúrgico.

\section{CASUÍSTICA E MÉTODO}

Nossa casuística é composta de 166 pacientes, registrados entre outubro de 1986 e dezembro de 1988, sendo os dados atualizados até fevereiro de 1992. Todos foram submetidos ao tratamento cirúrgico. 
O período mínimo de acompanhamento foi de 24 meses para 147 pacientes (média de 36 meses), sendo os demais 19 pacientes seguidos ambulatorialmente entre três e 18 meses.

As recomendações protocolares eram basicamente as seguintes:

- realizar quimioterapia pré-operatória apenas nos tumores julgados inicial mente irressecáveis;

- realizar incisão transversa ampla, verificar sinais de ruptura pré-operatória, sinais de disseminação e invasão de outros órgãos e anál ise sistemática do rim oposto, com abertura da fáscia de Gerota contralateral;

- avaliar possível extensão vascular da doença;

- tentar inicialmente ligar os elementos do pedículo renal;

- proceder à nefro-ureterectomia procurando-se evitar ao máximo a ruptura tumoral;

- avaliação minuciosa do comprometimento ganglionar, retirando ou biopsiando todo linfonodo hilar, periaórtico ou pericava;

- especificar a ocorrência e tipo de ruptura: Iocalizada na cápsula, com contaminação da Ioja

- renal ou de toda a cavidade peritoneal;

- especificar se a ressecção foi completa;

- tumores bilaterais: realizar biópsia bilateral e inventário ganglionar e, em seguida, a quimioterapia; ressecção pós-quimioterapia, procurandose a manutenção da mai or quantidade possível de parênquima renal, mesmo que para tal não se tenha adequada margem cirúrgica livre de tumor.

Tais dados foram submetidos a análi seestatística.

\section{RESULTADOS}

Nossos resultados têm por objetivo a análise estatística das variávei s dir etamente relacionadas à atuação do cirurgi ão no tratamento e no estadiamento do Tumor de Wilms. O resultado da análise das principais variáveis cirúrgicas é apresentado na tabela 1.

Quanto à influência do estádio clínico-cirúrgico-patológico na chance de recidiva e mortalidade observou-se que:

- a porcentagem de recidivas no estádio IV foi significantemente maior do que a verificada nos demais;

- a porcentagem de recidiva do estádio II foi menor do que a dos estádios I e III ;

- as porcentagens de recidivas dos estádios I e III não diferem entre si ;

- as porcentagens de óbitos dos estádios III e IV foram significantemente maiores do que as dos estádios I elI , sendo que I não difere de II , el II não difere de IV.
Os pacientes considerados no estádio $\mathrm{V}$ (tumor bilateral) não foram incluídos nesta análise estatística.

Em relação à análise do inventário ganglionar efetuado pelos cirurgiões, além da observação já referida na tabela 1 , de que os pacientes com gângli os comprometi dos tiveram um índice de recidivas menor do que os que não tiveram gânglios analisados, também verificamos que:

- existe associação entre os pacientes que não tiveram os gânglios retirados para análise e a maior chance de metástases pulmonares;

- a porcentagem de pacientes que tiveram gânglios retirados para a análise histopatológica foi significantemente maior nos casos em que o cirurgião teve também a preocupação de examinar o rim contralateral.

O estudo da concordância ou discordância entre a avaliação da gordura peri-renal efetuada pelo cirurgião da efetuada pelo patologista mostrou que em $15,8 \%$ dos casos o cirurgião errou ao julgar a gordura peri-renal não comprometida.

Finalmente, avaliamos a concordância ou discordância entre a avaliação da adrenal efetuada pelo cirurgião da efetuada pelo patologista, verificando que em $20,8 \%$ dos casos o cirurgião errou ao julgar a adrenal não comprometida.

\section{DISCUSSÃO}

Em uma equipe multidisciplinar, a primeira tarefa do cirurgião é participar da confirmação do diagnóstico e da decisão quanto à necessidade de realizar a quimioterapia pré-operatória, sendo que a filosofia do Grupo Cooperativo Brasileiro para o Tratamento do Tumor de Wilms é só empregar esse tratamento pré-operatório nos tumores considerados irressecáveis.

De uma forma indireta, neste estudo pode-se inferir que houve obediência a essa norma, pois, dado o alto índice de tumores volumosos nesta casuística (54\% superiores a 500 g e 17,7\% superiores a $1.000 \mathrm{~g}$ ), a quimi oterapia pré-operatória foi utilizada em $20 \%$ dos casos.

Em relação ao peso do tumor, observou-se pior prognóstico para os pacientes cujos tumores tinham peso igual ou superior a $500 \mathrm{~g}$. Esse achado foi também confirmado em estudo mais recente do GCBTTW ${ }^{1}$ e também no NWTS, pois se constitui em base para uma das recomendações do último estudo do NWTS, inaugurado em 1995, no qual é proposto tratamento exclusivamente cirúrgico para os paci entes no estádi o I, idade inferi or a dois anos e tumores cujo peso não ultrapasse $500 \mathrm{~g}^{2,3}$.

A obediência às normas do protocol o também foi 


\begin{tabular}{|c|c|c|}
\hline & \multicolumn{2}{|c|}{ Significância estatística } \\
\hline Variáveis & Recidiva & Mortalidade \\
\hline Ligadura prévia do pedículo & não-significante & não-significante \\
\hline Visualização do rim contralateral & não-significante & não-significante \\
\hline Ruptura pré-operatória & não-significante & não-significante \\
\hline Ruptura intra-operatória localizada & não-significante & não-significante \\
\hline Ruptura intra-operatória disseminada & *significante & *significante \\
\hline Invasão de vasos (v.renal e VCI) & não-significante & não-significante \\
\hline Invasão de estruturas vizinhas & não-significante & não-significante \\
\hline Peso do tumor (> $500 \mathrm{~g}$ ) & não-significante & *significante \\
\hline Inventário ganglionar & ${ }^{* *}$ significante & não-significante \\
\hline
\end{tabular}

verificada em relação à via de acesso, tendo sido quase todos os pacientes operados através de uma incisão transversa ampla. O mesmo se verificou quanto à tentativa de realizar a ligadura prévia dos el ementos do pedículo, efetuada em $54 \%$ dos casos, antes das manobras para a ressecção do tumor. No primeiro estudo do NWTS ${ }^{4}$ essa proporção foi maior (70\%), provavelmente devido ao maior número de tumores menores, fruto de uma maior proporção de diagnósticos precoces em centros mais desenvolvidos.

A exploração do rim contral ateral é recomendada tanto pelos pesquisadores da Sociedade Internacional de Oncologia Pediátrica $(\mathrm{SIOP})^{5}$ quanto pelos do NWTS ${ }^{6}$, sendo também uma recomendação do protocolo cirúrgico do GCBTTW.

Apesar dessa recomendação ser questionada para instituições que tenham ampla disponibilidade de recursos imagenológi $\cos ^{7}$, relato muito recente dos pesquisadores do NWTS ${ }^{8}$ refere que $7 \%$ dos tumores só serão diagnosticados durante a exploração cirúrgica. Em 32 (19,3\%) pacientes desta casuística, essa abordagem não foi efetuada, fato esse considerado uma violação às normas do protocolo estabelecido.

Foi ainda mais freqüente não realizar inventário ganglionar $(82,3 \%)$ entre os cirurgiões que não examinaram o rim contralateral em comparação aos demais (50,8\%). Essa diferença é estatisticamente significante, mostrando a existência de associação quanto ao não cumprimento de duas das principais normas de estadiamento do Tumor de Wilms, inventário gangli onar e expl oração do rim contralateral, em um grupo significante de pacientes, podendo-se daí concluir que esses pacientes foram estadiados inadequadamente.

A importância dessa verificação veio se confir- mar na continuação do estudo da casuística do GCBTTW, em 1996', sendo nessa ocasião a casuística composta por 602 pacientes. U ma das conclusões desse estudo foi a de que a não realização do exame do rim contralateral pode ser considerada um fator de aumento das chances de recidiva abdominal, independentemente do estadiamento.

Nesse mesmo estudo foi ainda verificada a importância da ruptura tumoral, de qualquer tipo, como fator de risco para óbito, sendo que no nosso estudo essa conclusão só havia si do verificada para os casos de ruptura com contaminação de toda a cavidade abdominal. As outras formas de ruptura não puderam ter confirmado seu risco real, provavelmente pelo número insuficiente de casos.

Outra discordância atribuível ao estadiamento cirúrgico foi a verificada em relação às avaliações cirúrgica e anatomopatológica da adrenal e da gordura peri-renal. Em 15,8 \% dos casos em que o cirurgião considerou a gordura peri-renal normal e em $20,8 \%$ em que ele considerou normal a adrenal, o patologista mostrou haver comprometimento neoplásico dessas estruturas. Com base nesses dados, podemos considerar que existe risco de subestadiamento do Tumor de Wilms na dependência dessa avaliação e esse risco seria teoricamente eliminado pela ressecção completa da gordura peri-renal em todos os casos e pela real ização de uma ou mais biópsias da adrenal, quando julgarmos não ser necessária a sua retirada em conjunto com o tumor.

E $m$ relação aos tumores bilaterais, o estudo da casuística do GCBTTW já foi motivo de publicação anterior ${ }^{10}$, não sendo aqui analisado.

O principal dado deste estudo é, ao nosso ver, aquel e que diz respei to à real ização do inventári o ganglionar, em que concluímos que o desempenho 
dos cirurgi ões ficou muito aquém do desejável em relação ao estabelecido no protocolo. A proporção de pacientes que não tiveram gângl ios analisados foi de $56,6 \%$ nessa casuística, valor muito el evado em comparação aos referidos na literatura, sendo que a proporção de gânglios não analisados no NWTS III foi de $11 \%{ }^{11}$. Lembramos que o comprometimento ganglionar é comprovadamente um dos princi pais fatores de pi ora do prognóstico nas avaliações dos pesquisadores do NWTS $2,9,11$, do grupo cooperativo do Reino Unido ${ }^{12}$ e da SIOP ${ }^{13}$.

Concluímos que esses dados podem estar contribuindo para a obtenção de resultados não esperados, tais como: menor índice de recidivas para os pacientes com gânglios acometidos e maior incidência de metástases pulmonares nos pacientes que não tiveram gânglios analisados em relação aos demais. Concluímos, ainda, que a inadequação do inventário ganglionar pode ter contribuído de forma desfavorável para a ocorrência de resultados não compatíveis com o estadiamento, tais como: pacientes no estádio II com melhor prognóstico em relação ao estádi o I e pacientes nos estádios I ell I com índice de reci divas semel hantes à análise estatística.

Nossa conclusão final é a de que verificamos a ocorrência de al gumas omissões, as quais comprometeram de forma significativa a acurácia do estadiamento cirúrgico. Dessa forma, nossa opinião é a de que, em nosso meio, o oncol ogista responsável pelo tratamento de portadores do Tumor de Wilms deva estabelecer uma estreita integração com uma equipe cirúrgica, cujos membros tenham o conhecimento preciso do papel do cirurgião, não somente no tocante ao tratamento cirúrgico, mas que sejam conhecedores dos mínimos detalhes do estadiamento dessa neoplasia e sua importância em relação à abordagem multidisciplinar.

\section{SUMMARY}

\section{The role of surgery in the treatment of Wilms Tumor}

Oвj ECTIVEs. To detect the effects of the surgical treatment and staging on the obtained results and to check the possible relationship between these results and the compliance, or not, with the preset surgical approach protocol (in accordance with the National Wilms Tumor Study-NWTS)..

MATERIAL AN METHOD. One hundred and sixty six operated on pati ents entered between October 1986 and December 1988, with the data updated until F ebruary 1992 were studi ed. Theminimum foll owup period was 24 months for 147 patients (average 36 months). The remaining 19 patients were fol- lowed in the outpatient clinic for three to 18 months.

Results: After submitting these data to statistical analysis and the obtained results compared to those in the literature we observed that:

-previous ligature of the renal vessels had no discernible effect on the ultimate outcome (relapses and mortality);

-intra-operative tumoral rupture with contamination of the peritoneal cavity unfavorably interferes with the mortality rate;

-even when theadrenal and the perirenal fat are normal from the surgeon's point of view, the histological findings showed tumor contamination in a number of cases;

-there is a worse prognosis when the tumor weight exceeds $500 \mathrm{~g}$;

-according to the Iymphonode evaluation, surgeon's performance was not in accordance with the protocol recommendations, considering that the node evaluation was neglected in $56.6 \%$ of the patients. This led to a surgical staging error and has contributed to some unexpected results like:

-lower relapses rate in the patients with affected Iymphnodes;

-higher incidence of lung metastases in patients without Iymphnodes analysis;

-stage II patients with better prognosis, compared to stage I patients and patients with stage I and III having similar relapse rates.

Concl usion: From the above-referred results, we concluded that some omissions have happened jeopardizing accuracy of the surgical staging in a significant way. In this way, in our environment the oncologist in charge of the treatment of Wilms tumor bearers, must stablish a close integration with a surgical team, whose members have a precise understanding of the surgeon's importance and his fundamental role, not only of the surgical treatment itself, but who areaware on the minimal details on the surgical staging of this neoplasm, and of its importance in relation to the multidisplinary approach. [Rev Ass Med Bras 1999; 45(4): 342-6]

KEY WORDS: Wilms Tumor. Nephroblastoma. Surgical treatment.

\section{REFERÊNCIAS BIBLIOGRÁFICAS}

1. DeCamargo, B. Fatores que influenciam o prognóstico clínico em pacientes com tumor de Wilms. Um estudo nacional. São Paulo, 1996. p. 179. Tese(Doutorado) - F aculdadedeM edicina da Universidade de São Paulo.

2. Green DM, Beckwith J B, Weeks DA et al. The relationship between the microsubstaging variables, age at diagnosis, and tumor weight of children with stage I / favorable histology Wilms' tumor: a report from the National Wilms' Tumor 
Study. Cancer, 1994; 74: 1817- 20.

3. Green DM, Breslow NE, Beckwith J B, et al. Treatment outcome in patients less than two years of age with small stagel, favorable histology, Wilms'tumors: a report from the National Wilms'Tumor Study. J . Clin. Oncol., 1993; 11: 91-95.

4. Leape LL, Breslow NE, Bishop HC. - The Surgical Treatment of Wilms'Tumor: results of the National Wilms Tumor Study. Ann. Surg., 1978; 187: 351-6.

5. Coopes MJ, De Kraker J, Van Dijken HJ M, Perry JFM, Tournade MF, LemerleJ , VoutePA.- Bilateral Wilms' tumor; long term survival and some epidemiological features. J . Clin. Oncol., 1985; 7: 310-5.

6. Blute ML; Kelalis PP; Offord KP; Breslow N; Beckwith J B; D'AngioGJ .- Bilateral Wilms'Tumor.J .U rol., 1987; 128: 968-73.

7. Koo AS; Martin AK; Hurvitz RS; Weese D; Applebaun R; F onkalsrud EW; Ehrlich RM.- The necessity of contralateral surgical exploration in Wilms'tumor with modern non invasive imaging techniques. J . Urol., 1990; 144: 416-7.

8. Ritchey ML, Green DM, Breslow NB et al. Accuracy of current imaging modalities in the diagnosis of synchronous bilateral
Wilms' tumor. A report from the National Wilms Tumor Study. Cancer, 1995; 75: 600-604.

9. Breslow NE, Churchil G, Beckwith J B, Fernbach DJ , Othersen HB, Teft M, D'Angio GJ. Prognosis for Wilms'tumor patients with non metastatic disease at diagnosis. Results of the Second National Wilms Tumor Study. J . Clin. Oncol., 1985; 3: 521-31.

10. Alfer, W, Assunção MC, Denes F - Partial nephrectomy for bilateral Wilms' tumor. Med. Pediat. Oncol., 1991; 19: 131-6.

11. Breslow NE, Sharples K, Beckwith J B, Takashima J , Kelalis PP, Green D, D'Angio GJ . Prognostic factors in non metastatic, favorable histology Wilms' tumor. Results of the Third National Wilms'Tumor Study. Cancer, 1991; 68: 2345-53.

12. Gough, DSC. Wilms' tumor and genitourinary rhabdomyossarcoma. Br. J . Urol., 1989; 63:109-116.

13. Lemerle J, Tournade MF, Gerard Marchand R, Flamant R, Sarrazin D, Flamant F, Lemerle M, J undt F, Zucker J M, Schweisguth O. Wilms'Tumor: natural history and prognostic factors. A retrospective study of 246 cases treated at the Institut GustaveRussy 1952-1967. Cancer, 1976; 37: 25-57-66. 